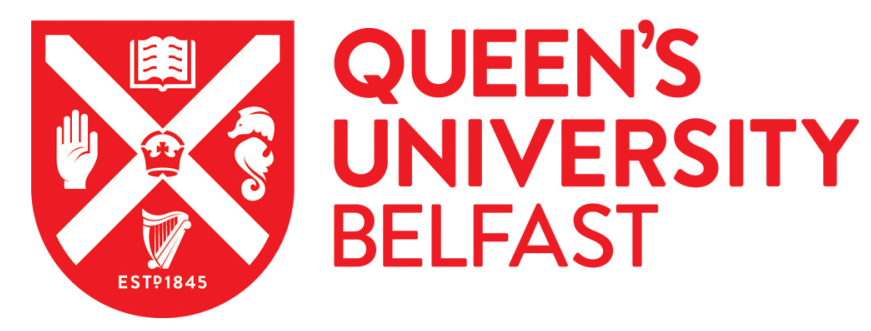

\title{
Generation of high contrast and high spatial quality idler from a low- gain optical parametric amplifier
}

Sharba, A. B., Nersisyan, G., Zepf, M., Stuart, N. H., Smith, R. A., Borghesi, M., \& Sarri, G. (2016). Generation of high contrast and high spatial quality idler from a low-gain optical parametric amplifier. Applied Optics, 55(33), 9341-9346. https://doi.org/10.1364/AO.55.009341

\section{Published in:}

Applied Optics

Document Version:

Peer reviewed version

Queen's University Belfast - Research Portal:

Link to publication record in Queen's University Belfast Research Portal

Publisher rights

(C) 2016 Optical Society of America. One print or electronic copy may be made for personal use only. Systematic reproduction and distribution, duplication of any material in this paper for a fee or for commercial purposes, or modifications of the content of this paper are prohibited

\section{General rights}

Copyright for the publications made accessible via the Queen's University Belfast Research Portal is retained by the author(s) and / or other copyright owners and it is a condition of accessing these publications that users recognise and abide by the legal requirements associated with these rights.

Take down policy

The Research Portal is Queen's institutional repository that provides access to Queen's research output. Every effort has been made to ensure that content in the Research Portal does not infringe any person's rights, or applicable UK laws. If you discover content in the Research Portal that you believe breaches copyright or violates any law, please contact openaccess@qub.ac.uk. 


\title{
Generation of High-Contrast and High-Spatial Quality Idler from a Low-Gain Optical Parametric Amplifier
}

\author{
A. B. Sharba ${ }^{1,2}$, G. Nersisyan ${ }^{1}$, M. ZePf ${ }^{1}$, N. H. Stuart ${ }^{3}$, R. A. Smith ${ }^{3}$, M. Borghesi ${ }^{1}$, And \\ G. SARRI ${ }^{1}$ \\ 1 Centre for Plasma Physics, School of Mathematics and Physics, Queen's University Belfast \\ 2Department of Laser Physics, College of Science for Women, Babylon University \\ 3Blackett laboratory, Department of Physics, Imperial College, London \\ *Corresponding author: asharba01@qub.ac.uk
}

\begin{abstract}
The temporal contrast of a regeneratively amplified, sub-picosecond pulse is enhanced employing a lowgain optical parametric amplification stage self-pumped by the second-harmonic of the pulse. Through careful characterization of the two related non-linear processes and optimization of the non-collinear geometry, a robust high-contrast idler pulse has been generated, with excellent spatial quality in both the near and far field. The overall energy conversion efficiency exceeds $14 \%$, with $33 \%$ intensity conversion efficiency. The temporal cleaning is implemented without any bandwidth losses or spectral shift and produces approximately $20 \%$ temporal shortening. These experimental findings are in excellent agreement with numerical calculations.
\end{abstract}

Exciting and rapidly developing investigations of laser-matter interactions at relativistic laser intensities have been enabled by the development of chirped-pulse amplification (CPA) techniques [1]. For these kinds of interactions the temporal contrast of the laser pulse is one of the key parameters, since prepulses and pedestals can damage or deform the target before the arrival of the main pulse $[2,3]$. A suitably high contrast for some applications cannot be achieved from a regenerative pre-amplifier because even a very small pre-pulse can be amplified to a level sufficient to pre-ionize the target. In addition, the amplified spontaneous emission (ASE) from regenerative amplifiers can form a long pedestal around the main pulse, contributing to the degradation of the temporal contrast. Therefore, the development of laser technologies enabling ever growing intensities has been accompanied by the development of many techniques [4-12] for enhancing the temporal contrast.

Short pulse, low gain optical parametric amplification (OPA) is one of the most efficient contrast enhancement techniques that can work at the front end of a high power laser system. Pumping an OPA with a sufficiently short pump pulse, typically shorter than $1 \mathrm{ps}$, ensures that the pump overlaps only with the main signal pulse, leaving discrete pre-pulses and the majority of the ASE pedestal outside the amplification window. This leads to enhancement of the temporal contrast of the signal by a factor equivalent to the gain factor [12]. Generating the pump pulse of the OPA by frequency doubling a part of the input pulse and using the idler rather than the signal can provide an extreme contrast enhancement that can reach up to the third power of the input contrast [9]. This theoretical prediction is limited only by parametric noise and scattered photons from the signal overlapping with the idler.

In its simplest configuration, as previously described by R. Shah, et al. [10], the contrast enhancement system consists of a second harmonic generation ( $\mathrm{SHG}$ ) and a non-collinear OPA stage. The input pulse is split into two parts and the doubling stage converts a large part of the pulse energy into a second harmonic (SH) pulse to pump the following OPA stage, which is seeded by the rest of the input pulse energy. The idler pulse is considered as the useful output of this system due to the high contrast of this pulse. 
In such a system, working with degenerate OPA requires a slight non-collinearity in order to spatially separate the idler and the signal. This configuration generally leads to the generation of an angularly dispersed idler beam due to conservation of momentum. Although the bandwidth of the generated idler is relatively small, a non-collinear angle of few degrees can result in significant spatial and temporal degradation in the generated pulse, see [Ref [13] section 3.2.4] for more details. Techniques such as pulse-front tilt [14] or post-amplification correction [15] could be used to correct the idler pulse-front shear. However, for pulses having relatively small bandwidth, none of these techniques are, in practice, the best solutions to the problem, due to the design complexity and the associated losses.

In addition, in such a system, using a crystal that is slightly shorter than the optimum length, which is defined by the intensity and the duration of the input pulse, limits the achievable conversion efficiency, while a crystal longer than the optimum length results in generation of spatially and/or temporally degraded idler pulses, as discussed in [13]. In addition, when the input pulse is non-transform limited, the spectrum of the generated idler can be shifted from that of the input pulse, [see e.g. fig (12) in Ref [13]].

In this work, we present experimental results including the generation of a high spatial quality and high contrast idler pulse with $>14 \%$ overall energy conversion efficiency and $>$ $33 \%$ intensity conversion efficiency, from an input pulse having about $190 \mu \mathrm{J}$ energy and 470 fs full-width half-maximum (FWHM) duration. The energy conversion efficiency is obtained by comparing the energy of the generated idler with that of the input pulse to the system, while the intensity efficiency is obtained by comparing the calculated intensities of the idler and the input beam to the system. The system efficiency and idler characteristics are optimized by using a crystal slightly longer than the calculated optimum thickness and introducing a suitable delay between the seed and the pump. By using this technique, the temporal cleaning can be implemented without any spectral shift or bandwidth losses. Using a small non-collinear angle in the OPA stage results in the generation of an idler beam having high spatial quality in both near and far field while preserving the capability of the system in generating pulses of high contrast. The temporal contrast of the generated idler is below the detection limit of the third-order auto-correlator employed $\left(\approx 7 \times 10^{-10}\right)$. The experimental results are in very good agreement with the numerical simulation presented in Ref [13].

The numerical simulation takes into account all the parameters at play in both of the related nonlinear processes. The spectral content of the pulse, group velocity mismatch $(\mathrm{GVM})$, the divergence of the beam, pump depletion and the crystal length were all considered into the simulation. The model used for simulating the results shown in this work, deals with the two related nonlinear processes as an integrated system where all of the characteristics of the pump pulse generated in the SHG stage are fed to the following OPA stage.

In comparison with the previously reported results by R. Shah, et al. [10], our work presents advancement represented by a $\sim 20 \%$ pulse shortening of the idler relative to the seed pulse, in contrast to the $12 \%$ bandwidth loss reported in the aforementioned work, for a comparable energy conversion efficiency. In addition, the spatial quality of the idler in the far field, and hence the achievable optical intensity, is improved in comparison to the case of using relatively large non-collinear angle, as will be discuss later. 
Figure (1) shows a layout of the temporal cleaning device described in this work, and its position at the front-end of the high-power laser system. A mode-locked oscillator (CoherentMira $900 \mathrm{~F}$ ) is used to generate 100 fs pulses at a nano-Joule energy level with $76 \mathrm{MHz}$ repetition rate. The oscillator pulse is stretched to $1 \mathrm{~ns}$ and then amplified to about $500 \mu \mathrm{J}$ in a $500 \mathrm{~Hz}$ repetition rate Ti-Shapphire regenerative amplifier. A two-grating, double-pass compressor is used to recompress the output to about 470 fs with efficiency $\sim 40 \%$. The output of the compressor, having $190 \mu \mathrm{J}$ energy and $5 \mathrm{~nm}$ bandwidth centred around 1053 $\mathrm{nm}$, seeds the high-contrast OPA. The contrast enhancement device consists of a 3:1 telescope, a 90/10 transmission/reflection beam splitter, and two $3 \mathrm{~mm}$ Beta Barium Borate (BBO) crystals, Type I phase matching. Two dielectric mirrors are used to separate the SH pulse from the unconverted fundamental harmonic (FH) after the SHG stage. The energy of the seed reaching the OPA crystal is $6 \%$ of the input energy, instead of $10 \%$, due to the losses of the mirrors located between the beam splitter and the OPA crystal. The seed-pump mixing in the OPA is carried out in the vertical plane to ensure that the direction of the angular dispersion of the idler will be in the lower sensitivity plane of the gratings in the stretcher and the compressor. This minimizes the influence of any residual angular dispersion of the idler on the subsequent stretch and the recompression processes.

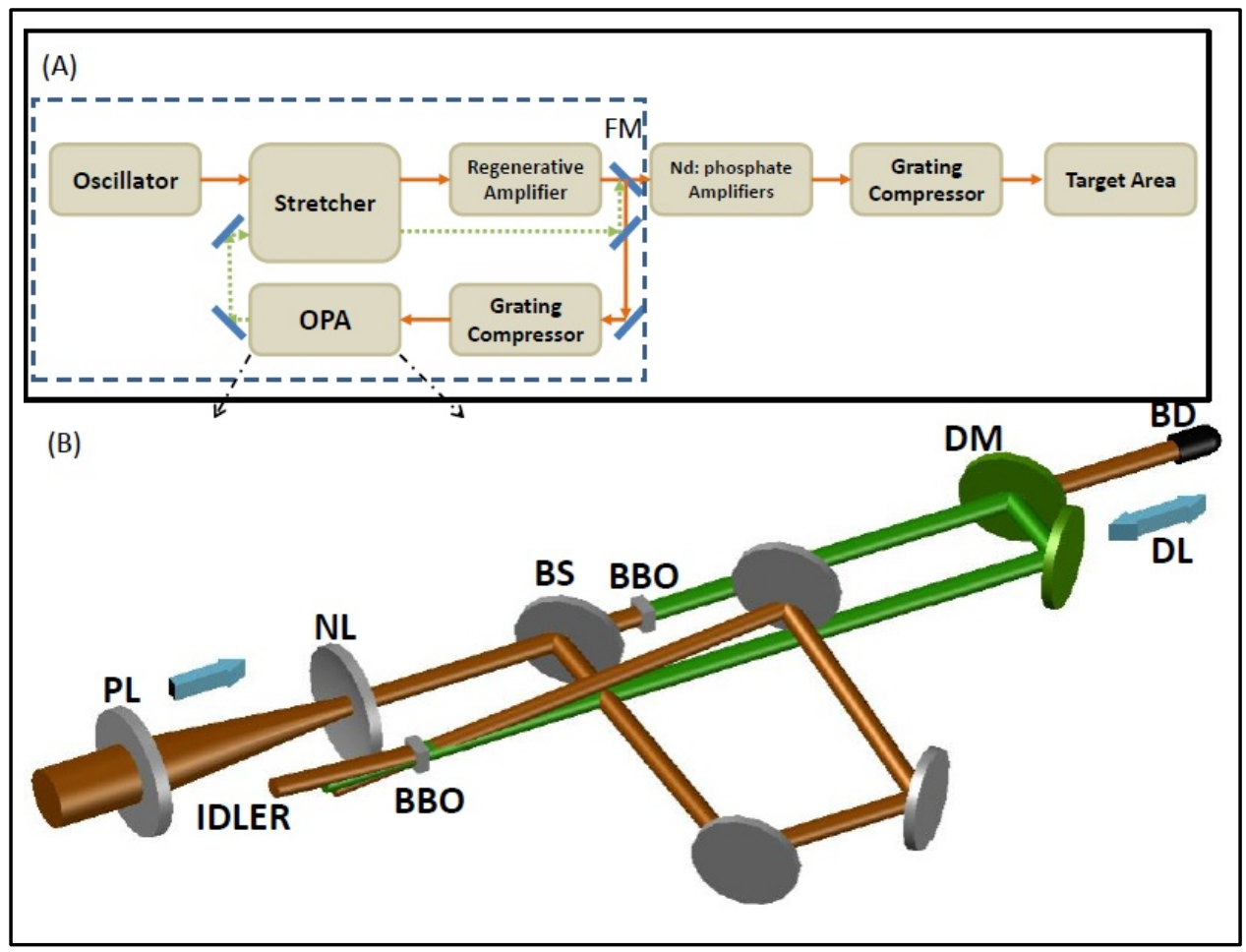

Fig. 1. (A) A simplified layout of the TARANIS laser system [16]. The front end of the system, the part in the dashed area, generates the input pulse for the high-contrast OPA. (B) A detailed layout of a SHG-OPA based temporal contrast enhancement system. FM: flip mirror, PL: positive lens, NL: negative lens, BS: beam splitter, DM: dielectric mirror, BD: beam dump and DL: delay line. The beam path for reinserting the high contrast idler to the laser chain (dote lines) is currently under development.

The intensity at the beam splitter, with reference to figure (1), is about $6.2 \mathrm{GW} / \mathrm{cm}^{2}$. The beam is then split, with the $90 \%$ component to be frequency doubled, which attains a maximum energy conversion efficiency that can exceed $65 \%$ from the $3 \mathrm{~mm}$ BBO crystal in the SHG stage. The SHG conversion efficiency in this case is limited by many factors such as the GVM, the limited acceptance bandwidth of the crystal and the divergence of the beam, as 
discussed in detail in our simulation presented in [13]. The generated SH pulse is mixed with the rest of the input energy in the following OPA stage at an external non-collinear angle $0.6^{\circ}$.

In the OPA stage, when the seed and the pump are perfectly synchronized, the pulse under study requires a crystal having a thickness about $2.1 \mathrm{~mm}$ for optimum conversion, according to the numerical simulation in Ref. [13]. The maximum obtainable energy conversion efficiency in the idler in this case is just $7.8 \%$, and $18 \%$ as intensity conversion efficiency. Also, in this case, since the input pulse is a non-transform limited pulse, the idler spectrum will be shifted from the input spectrum, which is not desirable in terms of matching the amplification wavelength of the laser chain. However, by using a $3 \mathrm{~mm} \mathrm{BBO}$ crystal and delaying the seed by about 300 fs relative to the pump, the overall energy conversion efficiency of the system, into the idler, is almost doubled and the intensity conversion efficiency is raised up to $33 \%$. In this case, delaying the seed minimizes the effect of GVM, and hence results in the generation of an idler pulse having a pulse duration of about $370 \mathrm{fs}$ ( $\pm 5 \mathrm{fs})$, as shown in figure ( $2 \mathrm{~A})$, while the idler pulse that is generated at perfect temporal superposition would have a $545 \mathrm{fs}$ duration at the exit face of the $3 \mathrm{~mm}$ crystal.

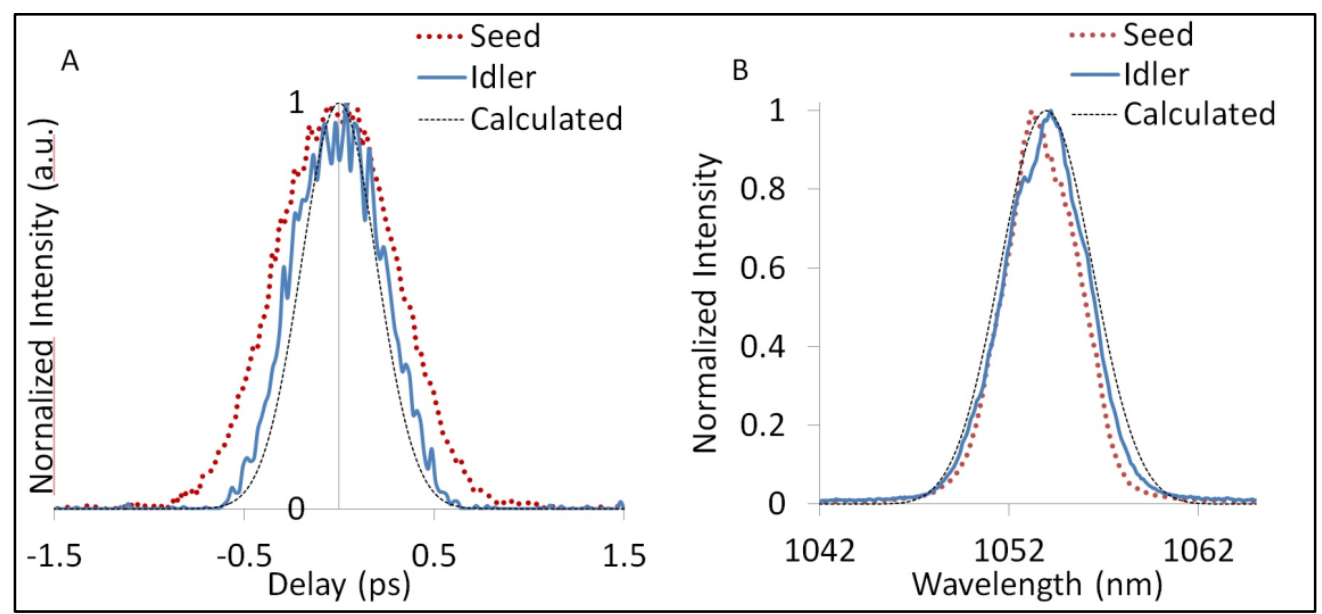

Fig. 2. A: The simulated and the measured second-order autocorrelation traces for an idler pulse that is generated in a $3 \mathrm{~mm} \mathrm{BBO}$ from a $470 \mathrm{fs}$ input pulse to the system, B: the spectra of the input pulse and the generated idler along with a simulated idler spectrum.

In such a configuration, the delayed seed pulse, which has higher group velocity, will scan the slower pump pulse as they both propagate through the crystal, while in the case of initially matched pulses, the seed and the idler would walk away from the pump at a speed depending on the difference between their group velocities. Compared to this case, the main advantages of our configuration are: first, the seed, and hence the idler, will gain more energy from the pump as they scan the pump pulse, which enhances the conversion efficiency of the process. Second, generating the idler in the seed-pump overlapped region, which is in this case increasing as the pulses propagate through the crystal, results in decreasing the GVMinduced broadening of the idler pulse. Hence delaying the faster pulse leads to the generation of a shorter idler pulse, with broader bandwidth, than in case of initially matched seed-pump pulses. A discussion providing more details about this effect can be found in [13] and references therein.

Moreover, besides maximizing the conversion efficiency and generating a shorter idler pulse, delaying the seed in a longer crystal compensates for the idler spectral shift and brings 
it back to match the input spectrum, as shown in frame B of figure (2). It is clear that the idler pulse in this case retains the same input bandwidth without any spectral losses.

In order to study the response of the system, the energy of the input pulse was attenuated using a wave-plate and polarizer. The energy conversion efficiency of the frequency doubling stage as a function of the energy of the incident beam on the doubling crystal is shown in figure ( $3 \mathrm{~A})$, along with the simulated curve. At input energies around the maximum, the doubling efficiency shows saturation of the process. In this regime, besides achieving good conversion stability, the beam size of the generated SH increases to almost match that of the incident beam. This results in maximizing the conversion efficiency in the following OPA stage and increases the idler beam size.

Figure (3 B) presents the variation of the overall energy conversion efficiency of the system into the idler pulse as a function of the total input energy. The results in the figure have been obtained by attenuating the total input energy to the system without changing the delay in the OPA after it has been optimized at the maximum input energy. The excellent agreement between the experimental and the simulated results support the validity of the model at predicting the performance of such a system.

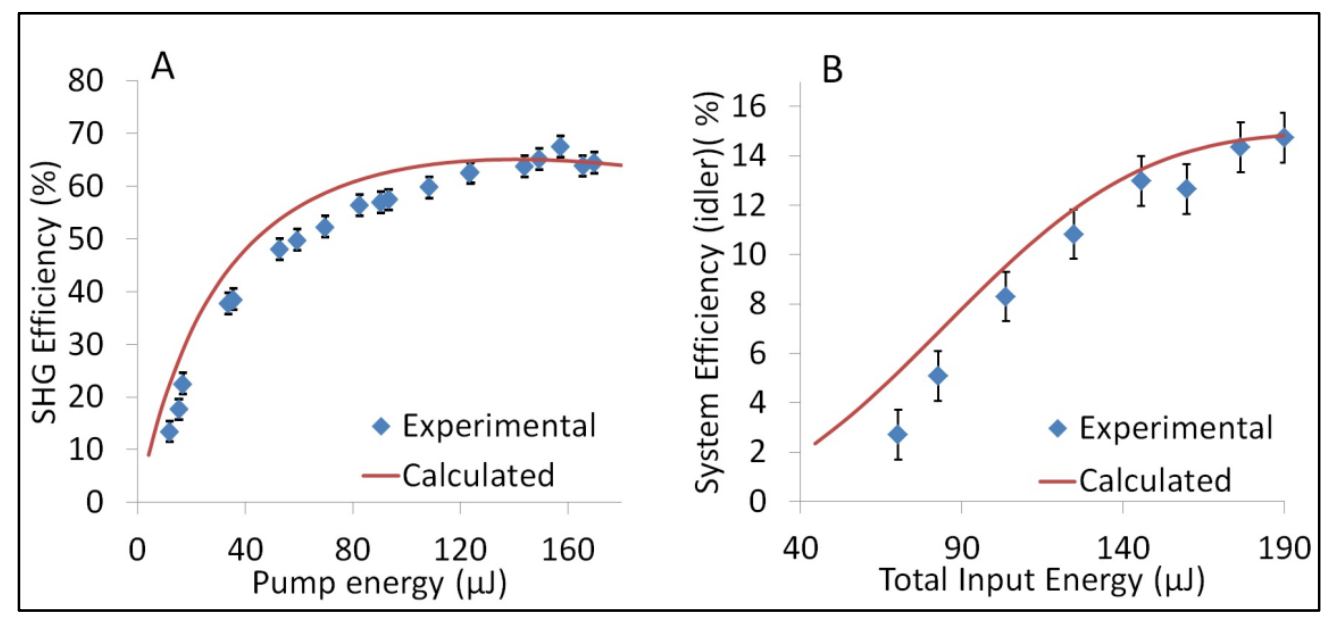

Fig. 3. A: The calculated and the experimental SHG efficiency as a function of the energy of the incident pulse on the SHG crystal, B: the total energy conversion efficiency into the idler as a function of the total input energy.

The energy conversion efficiency in the OPA stage in this case, by comparing the idler energy to the pump energy, is about $23.4 \%$. This efficiency is mainly limited by three factors: the intensity of the pump pulse, which is generated in the SHG stage, the bandwidth of the input pulse, and the GVM between the amplified and pump pulse. The effect of the divergence of the interacting beams on the OPA conversion efficiency, in case of unfocused beams, is found to be very small. However, the overall conversion efficiency of such a system is defined, (in addition to the spectral content of the pulse, the GVM, the divergence of the beam and the configuration of the apparatus), by any residual chirp on the pulse to be cleaned. A better compression results in enhancing the overall performance of the system.

The spatial profile and the quality of the generated idler beam were investigated in the near and far field for two different non-collinear angles, $3.7^{\circ}$ and $0.6^{\circ}$ (external). In both cases the idler beam shows a circular shape in the near-field, with about $1.35 \times$ reduction in beam size relative to the initial input beam. An almost identical result has been obtained in [10], with $1.5 \times$ reduction in an idler beam that is generated with a $4^{\circ}$ external non-collinear angle. 
Figure (4 A) presents an image in the nearfield for an idler beam generated using an external non-collinear angle $0.6^{\circ}$. The profile of the image is shown in frame (D) of the figure.

However, the spatial profile of the idler beam in the far field strongly depends on the mixing angle in the OPA stage. This is because the angular dispersion, which depends on the mixing angle, leads to an enlargement in the beam focus due to the spectral separation in the mixing dimension [17]. This can be seen clearly in figures (4 B and E), which show the focal spot and the profiles of an idler beam generated using a non-collinear angle of $3.7^{\circ}$. Given that the seed has a perfectly circular focal spot with size $\sim 1.2 \times$ the diffraction-limit (DL), using this angle leads to an enlargement of the idler focus up to $\sim 1.9 \times$ DL in the mixing dimension, while the perpendicular dimension retains the same quality of the input beam. This results in the formation of an elliptical focal spot of ellipticity $\sim 0.62$. For instance, assuming no local bandwidth reduction, the focus enlargement in this case along with the accompanying temporal broadening, due to the wave front tilt, simply results in the reduction by a factor 4 of the optical intensity in the focal plane for a beam of $\sim 2.2 \mathrm{~mm}$ FWHM. Using the same non-collinear angle, this effect is increased by two factors; the bandwidth and the beam size of the generated idler. The scaling of the intensity reduction in the focal plane as a function of the non-collinear angle is shown in more detail in Ref. [13], figure (18).

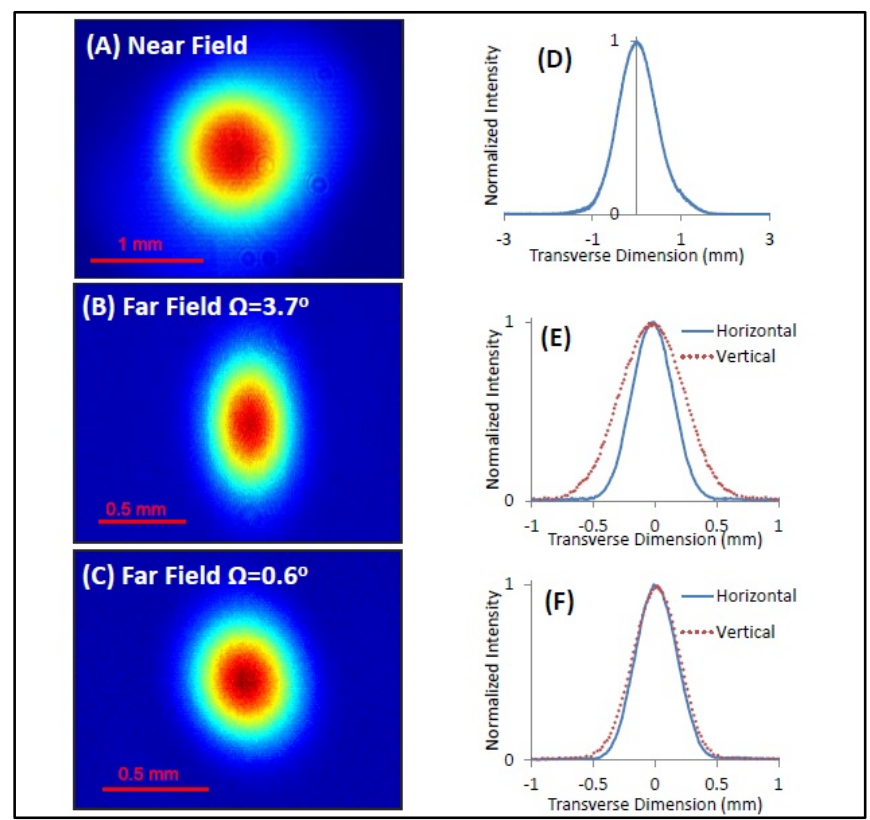

Fig. 4. A \& D The near-field of an idler beam that is generated with a 0.6 external non-collinear angle and its profile, respectively, B \& C: the focal spot of idler beams that are generated with non-collinear angle $\mathrm{W}=3.7_{-}$and $\mathrm{W}=0.6_{-}$, respectively, a $1 \mathrm{~m}$ focal length lens is used for focusing, $\mathrm{E} \& \mathrm{~F}$ the spatial profiles in the vertical and the horizontal directions of the images in $\mathrm{B}$ and $\mathrm{C}$, respectively.

Despite the advantage of using a large non-collinear angle in minimizing the level of the photons scattered from the signal to the idler [18], the resulting idler cannot be practically used as the seed of a high power laser system due to the angular dispersion. For a pulse of relatively small bandwidth, pre or post amplification techniques for dispersion correction are not practical, due to their complexity and inherent losses. Alternatively, since the unit is designed to work at an energy level where the use of small beams is possible, a small seed- 
pump mixing angle can be used in a compact footprint without degrading the idler temporal contrast. To demonstrate this, a $0.6^{\circ}$ non-collinear angle has been used in the OPA stage. At this angle, the size of the idler focal spot in the mixing direction is reduced to $<1.3 \times$ DL, i.e. $<0.1$ DL increment relative to the seed, while retaining the same quality of the input beam in the perpendicular direction. This reduces the ellipticity of the focus to about 0.9 . Figures (4 $\mathrm{C}$ and $\mathrm{F}$ ) show the focal spot and the spatial profiles in both directions for an idler beam that is generated using a $0.6^{\circ}$ non-collinear angle respectively. The optical intensity at the focus in this case reaches $>93 \%$ of the intensity of the same idler beam but without angular dispersion. Similar results, in terms of the spatial quality, have been obtained using $4 \times$ and 8 $\times$ smaller F-number focusing optics. This means that the unit does not significantly affect the beam quality after temporal cleaning.

In such a system the use of a small non-collinear angle is unlikely to significantly influence the conversion efficiency of the OPA process. This is because the effect of GVM, which can be reduced by using a particular relatively large angle, is compensated for by the pre-delay of the seed. In addition, since the OPA crystal is much shorter than the spatial walk-off length of the pump, the use of any non-collinear angle is not expected to induce any enhancement in the conversion efficiency via matching the direction of energy flow of the pump and one of the amplified beams.

In terms of the temporal contrast of the generated idler pulse, two measurements were recorded to investigate the contrast enhancement achieved. First, the level of the scattered photons in the idler line has been measured using a fast diode and a $1 \mathrm{~m}$ focal length lens to collect the light on the diode. The level of the scattered photons from the signal to the idler with pump-blocked is $2.7 \times 10^{-8}$ relative to the idler intensity with pump enabled. Hence, for an input seed pulse having a contrast ratio $10^{-5}$, the scattered photons will be at $\sim 10^{-13}$ level. This experimentally demonstrates that the contrast of the idler pulse will not be degraded by the scattered photons from the signal. Second, a high dynamic range third-order autocorrelator developed by the authors [19] has been used to measure the contrast of the seed and the idler pulses in the pico-second scale with 210 fs resolution. Given that the ASE level of the input pulse exceeds the $10^{-5}$ level in the pico-second scale, figure (5) shows that the cleaning system reduces that level below the detection-threshold of the third order autocorrelator $\left(\approx 7 \times 10^{-10}\right)$. The high contrast ratio of the generated pulse along with the high spatial quality demonstrate the potential of the system at cleaning the front end seed of a high power laser without adding any degradation to the seed.

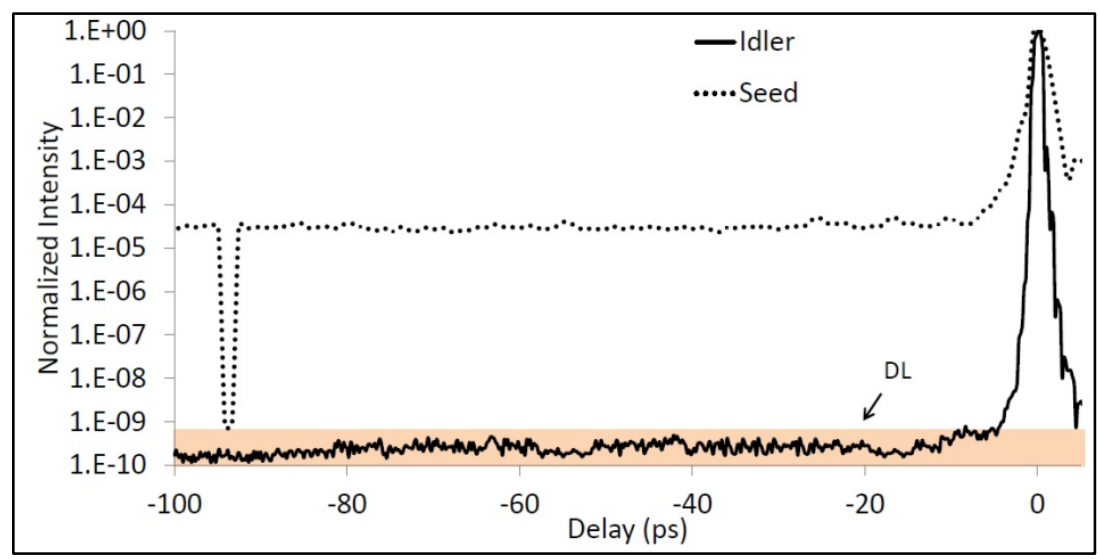

Fig. 5. Measured third order autocorrelation traces of the input seed pulse and the generated idler pulse. The cut off on the trace of the input pulse is obtained by blocking the input beam to the auotocorrelator in order to check the noise level. DL: is the detection limit. 
The high contrast of the idler is further confirmed by implementing a contrast measurement on the nanosecond scale using a fast diode and a Pockel cell, similar to the measurement configuration used in [12]. As shown in Fig. (6), the ASE and all of the prepulses on the output pulse of the regenerative amplifier have been suppressed to a level less than the noise level of the diode. The contrast measurement in this case is limited by the available energy of the idler.

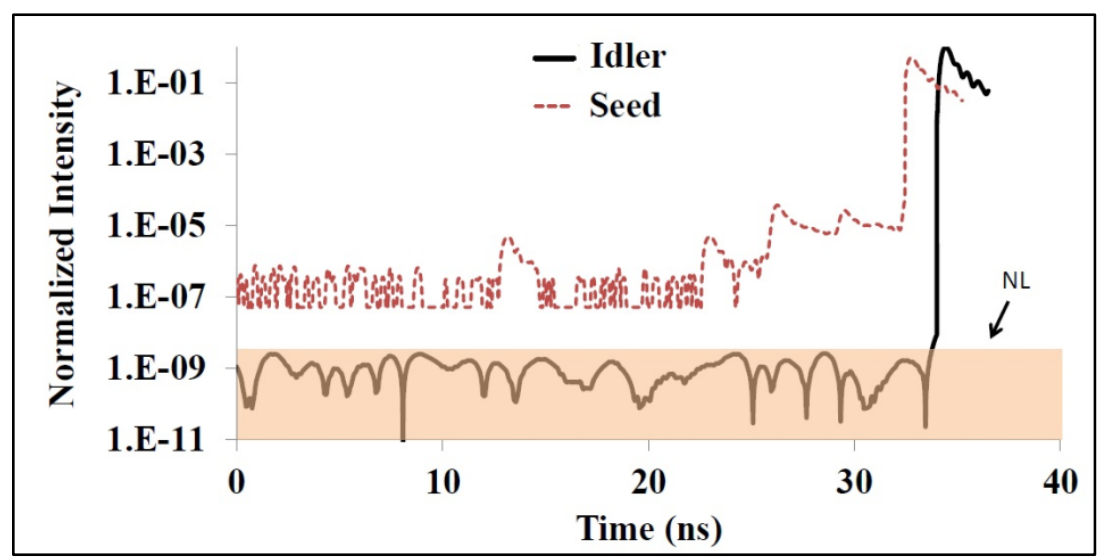

Fig. 6. Measured contrast of the seed and the generated idler using a fast diode and a Pockel cell. The measurements are shifted in time for clarity. NL: is the noise level of the diode.

The relatively low contrast ratio of the regenerative amplifier in both of the contrast measurements, Fig. (5 and 6), can be due to two reasons. First, although the pulse of our oscillator has a high contrast ratio, as reported in [19], the low available energy from the oscillator at the time of implementing the contrast measurement forced us to increase the number of passes into the regenerative amplifier, seeking more gain. This could lead to an increase of the ASE level in the output pulse. Second, for the purpose of demonstrating the robustness of our OPA system at enhancing the contrast, the last Pockel cell, which is installed to enhance the contrast on the nanosecond scale, was kept open for a longer time window, i.e. allowing the full ASE pedestal to be with the output pulse. However, in either case, the idler retains a very high contrast level that can significantly improve the contrast of the high power laser system.

In summary, we report on the generation of a highly optimized idler pulse using a low-gain optical parametric amplification technique as a means of temporal contrast enhancement. By using a small non-collinear angle and a carefully characterized system, a high contrast and high quality idler beam, in both the near and far field, is generated. The overall energy and intensity conversion efficiencies of the unit can exceed $14 \%$ and $33 \%$ respectively. The temporal contrast of the transmitted pulse has been enhanced from $\sim 10^{-5}$ to a value below the detection threshold of the third-order auto-correlator employed $\left(\approx 7 \times 10^{-10}\right)$. The high contrast of the idler pulse extends to within less than $10 \mathrm{ps}$ before the main peak. The cleaning is done without any bandwidth losses or spectral shift and the generated idler pulse is about $100 \mathrm{fs}$ shorter than the input seed. Due to the small non-collinear angle used, the spatial quality of the input beam is maintained during the temporal contrast enhancement in both the near and far field with negligible effects from angular dispersion. The good agreement between the experiment and the predicted results from simulations supports the validity of the model at 
characterizing such a system and allows estimation of the required parameters for optimum performance.

Funding. Engineering and Physical Sciences Research Council (EPSRC) (grant numbers EP/I029206/1 and EP/L013975/1) and the Iraqi Ministry of Higher Education and Scientific Research.

Acknowledgements. We gratefully acknowledge the assistance of the Center of Plasma Physics staff at Queen's University Belfast.

\section{REFERENCES}

1. D. Strickland and G. Mourou, "Compression of amplified chirped optical pulses," Opt. Commun. 56, 219-221 (1985).

2. D. Batani, R. Jafer, R. Redaelli, R. Dezulian, O. Lundh, F. Lindau, A. Persson, K. Osvay, C.-G. Wahlström, D. C. Carroll, P. McKenna, A. Flacco, V. Malka, and M. Veltcheva, "Effects of laser prepulse on proton generation," Nuclear Instruments and Methodsin Physics Research A 620, 76-82 (2010).

3. A. Andreev, R. Sonobe, S. Kawata, S. Miyazaki, K. Sakai, K. Miyauchi, T. Kikuchi, K. Platonov, and K. Nemoto, "Effect of a laser prepulse on fast ion generation in the interaction of ultra-short intense laser pulses with a limited-mass foil target," Plasma Phys. Control. Fusion 48, 1605 (2006).

4. V. Chvykov, P. Rousseau, S. Reed, G. Kalinchenko, and V. Yanovsky, "Generation of 1011 contrast 50 tw laser pulses," Opt. Lett. 31, 1456-1458 (2006).

5. D. Homoelle, A. L. Gaeta, V. Yanovsky, and G. Mourou, "Pulse contrast enhancement of high-energy pulses by use of a gas-filled hollow waveguide," Opt. Lett. 27, 1646-1648 (2002).

6. C. Zhang, J. Wang, C. Li, X. Chen, Y. Leng, L. Lin, R. Li, and Z. Xu, "Pulse temporal cleaner based on nonlinear ellipse rotation by using BK7 glass plate," Chin. Phys. Lett. 25, 2504 (2008).

7. J. Itatani, J. Faure, M. Nantel, G. Mourou, and S. Watanabe, "Suppression of the amplified spontaneous emission in chirped-pulseamplification lasers by clean high-energy seedpulse injection," Opt. Commun. 148, 70-74 (1998).

8. M. Kalashnikov, E. Risse, H. Schönnagel, and W. Sandner, "Double chirped-pulseamplification laser: a way to clean pulses temporally," Opt. Lett. 30, 923-925 (2005).

9. Y. Wang and B. Luther-Davies, "Optical-parametric-amplification-based prepulse eliminator for a chirped-pulse-amplification Nd: glass laser,” J. Opt. Soc. Am. B 11, 1531-1538 (1994).

10. R. C. Shah, R. P. Johnson, T. Shimada, K. A. Flippo, J. C. Fernandez, and B. M. Hegelich, "High-temporal contrast using low-gain optical parametric amplification," Opt. Lett. 34, 2273-2275 (2009).

11. C. Thaury, F. Quéré, J.-P. Geindre, A. Levy, T. Ceccotti, P. Monot, M. Bougeard, F. Réau, P. d'Oliveira, P. Audebert, R. Marjoribnks, and P. Martin, "Plasma mirrors for ultrahigh-intensity optics," Nature Phys. 3, 424-429 (2007).

12. I. Jovanovic, C. Barty, C. Haefner, and B. Wattellier, "Optical switching and contrast enhancement in intense laser systems by cascaded optical parametric amplification," Opt. Lett. 31, 787-789 (2006).

13. A. Sharba, G. Nersisyan, M. Zepf, M. Borghesi, and G. Sarri, "Comprehensive numerical modelling of the performance of a second harmonic generation stage coupled with a lowgain optical parametric amplifier," Opt. Express 24, 5212-5234 (2016). 
14. S.-W. Huang, J. Moses, and F. X. Kärtner, "Broadband noncollinear optical parametric amplification without angularly dispersed idler," Opt. Lett. 37, 2796-2798 (2012).

15. A. Shirakawa, I. Sakane, and T. Kobayashi, "Pulse-front-matched optical parametric amplification for sub-10-fs pulse generation tunable in the visible and near infrared," Opt. Lett. 23, 1292-1294 (1998).

16. T. Dzelzainis, G. Nersisyan, D. Riley, L. Romagnani, H. Ahmed, A. Bigongiari, M. Borghesi, D. Doria, B. Dromey, M. Makita, S. White, S. Kar, D. Marlow, B. Ramakrishna, G. Sarri, M. Zaka-Ul-Islam, M. Zepf, and C. L. S. Lewis, "The TARANIS laser: A multiterawatt system for laser-plasma investigations," Laser Part. Beams 28, 451-461 (2010).

17. G. Pretzler, A. Kasper, and K. Witte, "Angular chirp and tilted light pulses in CPA lasers," Appl. Phys. B 70, 1-9 (2000).

18. E. J. Divall and I. N. Ross, "High dynamic range contrast measurements by use of an optical parametric amplifier correlator," Opt. Lett 29, 2273-2275 (2004).

19. N. Stuart, T. Robinson, D. Hillier, N. Hopps, B. Parry, I. Musgrave, G. Nersisyan, A. Sharba, M. Zepf, and R. A. Smith, "Comparative study on the temporal contrast of femtosecond mode-locked laser oscillators," Opt. Lett. 41, 3221-3224 (2016). 